\title{
COMBINED FIRST AND SECOND ORDER FERMI ACCELERATION AT COMETS
}

\author{
T. I. Gombosi, ${ }^{*}$ K. Lorencz ${ }^{* *}$ and J. R. Jokipii*** \\ * Space Physics Research Laboratory, Department of Atmospheric, Oceanic \\ and Space Sciences, The University of Michigan, Ann Arbor, Michigan \\ 48109, U.S.A. \\ ** Space Physics Research Laboratory, Department of Atmospheric, Oceanic \\ and Space Sciences, The University of Michigan, Ann Arbor, Michigan \\ 48109, U.S.A. and Central Research Institute for Physics, Hungarian \\ Academy of Sciences, Budapest, P.O. Box 49, Hungary 1525 \\ ***Departments of Astronomy and Planetary Sciences, The University of \\ Arizona, Tucson, Arizona 85721, U.S.A.
}

\section{ABSTRACT}

Our two-dimensional, time-dependent model calculations indicate that an interplay between velocity and spatial diffusion may be responsible for the acceleration of implanted heavy ions in the cometary preshock region. Velocity diffusion (second order Fermi acceleration) accelerates the pickup ions to moderate energies thus creating a seed population for the more efficient diffusivecompressive shock acceleration. Solar wind convection limits the time available for diffusive-compressive acceleration, therefore the resulting energy spectrum above $100 \mathrm{keV}$ is a power law with a spectral index of $5-6$, a value which is in reasonably good agreement with observations.

\section{INTRODUCTION}

The interaction of the solar wind with a comet results in a variety of phenomena which have been observed in fly-bys of the comets Giacobini-Zinner and Halley. Of considerable interest is the acceleration of recently ionized cometary particles which are picked up by the solar wind and which form a superthermal particle population which can be accelerated by processes occurring in the cometary upstream region. This problem has been first examined in a pioneering paper by Amata and Formisano /1/, published well before the Giacobini-Zinner encounter. In a subsequent paper written shortly before the Halley encounters Ip and Axford /2/ considered five potential mechanisms which can act to accelerate the implanted ions. They argue that, because the shock in the vicinity of the comet was so weak $(\mathrm{M}=2)$, due to the contimuous mass loading of the solar wind, the first-order Fermi acceleration at the shock would be very slow. They concluded that in cometary environments the second-order Fermi acceleration would be the dominant mechanism.

It has recently been recognized that the second-order Fermi mechanism may have difficulties in explaining the observed acceleration of implanted heavy cometary ions to energies exceeding $\sim 250 \mathrm{keV} / 3,4 \%$. It should be noted that even though the second-order Fermi acceleration is probably important at comet Halley where the large gas production rate $\left(-10^{-30}\right.$ molecules/s at the time of the encounters) resulted in a very extended upstream pick-up region with significant levels of wave activity, in the case of comet Giacobini-Zinner the significantly smaller gas production rate was probably insufficient for this stochastic acceleration to operate effectively.

In an earlier paper we suggested that a combination of second-order Fermi acceleration and diffusive-compressive acceleration may be responsible for the acceleration of energetic particles in cometary environments $/ 5 /$. This paper presents the results of a two-dimensional (one spatial and one velocity space) time-dependent calculation and demonstrates that under quite reasonable circumstances, the entire foreshock region (where the solar wind slows down from a speed of roughly $500 \mathrm{~km} / \mathrm{s}$ to a couple of hundred kilometers per second) can serve as a region of a diffusive-compressive acceleration of energetic ions. This means that one has effectively a strong shock $(M=13)$ to energize particles and not only a weak subshock $(M=2)$ as was considered by Ip and Axford 21 . The requirement for this to occur is that the diffusion coefficient be, on the one hand large enough so that the entire foreshock region is seen as a "shock", but on the other hand, that it be small enough that the acceleration time scales are short enough to be relevant. We feel that these conditions can be satisfied for implanted ions in the foreshock region of strong shocks (such as the ones seen at comets Halley and Giacobini-Zinner), and suggest that in the cometary foreshock region firstorder Fermi acceleration may be an important energization mechanism of pick-up ions. At the same time it is also shown that second-order Fermi acceleration operates efficiently in the upstream region of comet Halley (the region where there is enough mass loading to generate MHD waves but the plasma flow does not show significant deceleration, yet) as suggested by Ip and Axford $2 \%$.

\section{MODEL}

Mass loading of the upstream solar wind flow starts at very large distances from an active comet. For instance in the case of comet Halley the first signatures of implanted ions were detected more than 10 million kilometers before closest approach $/ 6 /$. On the other hand no significant decrease of the flow Mach number was observed till about two million kilometers. Model calculations using parameter values characterizing comet Halley conditions during the spacecraft encounters $/ 3 /$ indicate that along the subsolar flow line the Mach number starts to decrease from its upstream value of -13 around $10^{6} \mathrm{~km}$ and reaches the $M=2$ value at around $3.5 \times 10^{5} \mathrm{~km}$. At this distance the development of a weak shock is predicted $\Pi /$ and the flow becomes subsonic. In the present paper the cometary foreshock is defined as the region where the flow velocity rapidly decreases, i.e. between $x_{1}=10^{5}$ and $x_{2}=3.5 \times 10^{5}$ kilometers. The characteristic length of this foreshock is $L \approx 5 \times 10^{5} \mathrm{~km}$. For the sake of mathematical simplicity a parallet shock geometry is assumed (see Fig. 1.) and perpendicular diffusion is neglected. 


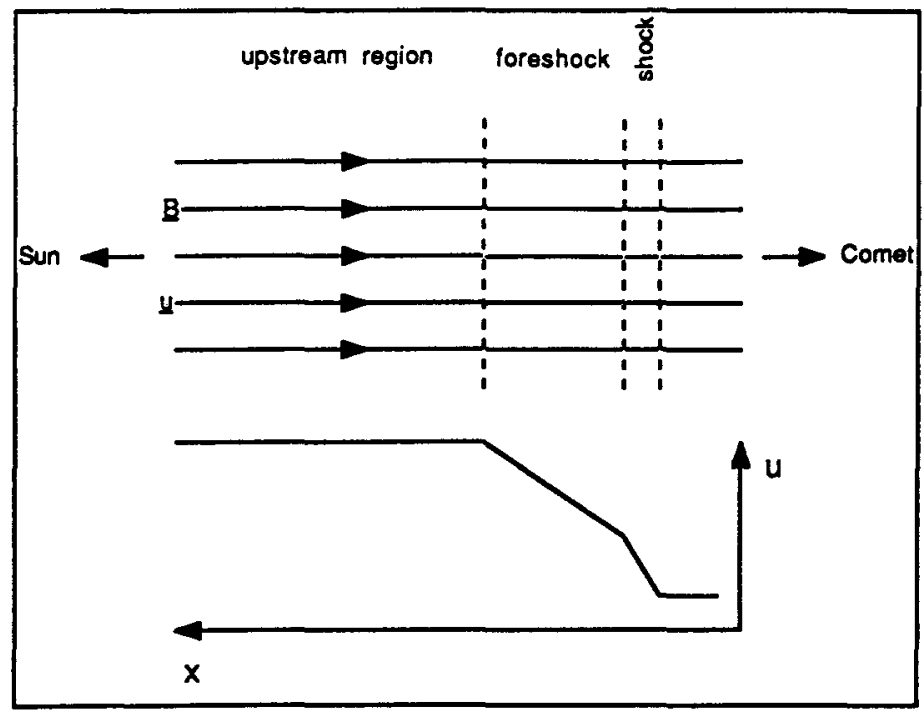

Fig. 1. Schematic representation of the subsolar cometary upstream region. The plasma flow deceleration starts at about $10^{6} \mathrm{~km}$, and a weak shock $\left(5 \times 10^{4} \mathrm{~km}\right.$ wide) is formed at $3.5 \times 10^{5} \mathrm{~km}$ from the comet.

It is assumed that energetic particles of cometary origin are primarily implanted $\mathrm{O}^{+}$ions. The velocity distribution of these ions is assumed to be near isotropic in the decelerating solar wind frame because of the rapid pitch-angle scattering by magnetic field turbulence. In this diffusion limit the particle distribution function, F, satisfies the following cosmic ray transport equation (cf. (3,8):

$$
\frac{\partial F}{\partial t}+u \frac{\partial F}{\partial x}-\frac{v}{3} \frac{d u}{d x} \frac{\partial F}{\partial v}=\frac{1}{v^{2}} \frac{\partial}{\partial v}\left(v^{2} D \frac{\partial F}{\partial v}\right)+\frac{\partial}{\partial x}\left(k \frac{\partial F}{\partial x}\right)+\frac{Q_{n} e^{-\frac{p}{\lambda}} \delta(u-v)}{16 \pi^{2} \lambda_{n} v^{2} r^{2}}
$$

Here $t$ is time, $x$ is distance along the flow line ( $x$ increases towards the comet), $r$ is cometocentric distance, $v$ is implanted ion velocity in the plasma frame, $u$ is plasma bulk velocity, $D$ is velocity diffusion coefficient, $K$ is spatial diffusion coefficient, $Q_{n}$ is cometary gas production rate, and $\lambda_{\eta}$ is the ionization scalelength of cometary oxygen. $F(x, v, t)$ is the pitch angle averaged phase-space density of oxygen ions having random velocity magnitude $v$ at time $t$ and position $x$. Introducing a modified distribution function, $f=4 \pi v^{2} F$, equation (1) can be expressed in the following form:

$$
\frac{\partial f}{\partial t}=D \frac{\partial^{2} f}{\partial v^{2}}+\left(\frac{v}{3} \frac{d u}{d x}-\frac{2 D}{v}+\frac{\partial D}{\partial v}\right) \frac{\partial f}{\partial v}+\left(\frac{2 D}{v^{2}}-\frac{2}{v} \frac{\partial D}{\partial v}-\frac{2}{3} \frac{d u}{d x}\right) f+k \frac{\partial^{2} f}{\partial x^{2}}+\left(\frac{\partial K}{\partial x}-u\right) \frac{\partial f}{\partial x}+\frac{Q_{n} e^{-\frac{r}{\lambda_{n}}} \delta(u-v)}{4 \pi \lambda_{n} r^{2}}
$$

A simple expression for $D$ was introduced earlier by Fisk $19 /$, who assumed a second-order Fermi acceleration process caused by interaction with propagating Alfven waves:

$$
\mathrm{D}=\frac{3}{4} \mathrm{~V}_{\mathrm{A}}^{2} \frac{\mathrm{v}}{\lambda}
$$

where $\lambda$ is the scattering mean free path, while the Alfven velocity, $v_{A}$, can be expressed as

$$
V_{A}^{2}=\frac{B^{2}}{4 \pi \rho_{i o n}}
$$

where $B$ is the magnetic field magnitude and $p_{i o n}$ is the total ion mass density. A similarly simple expression can be obtained for the spatial diffusion coefficient (cf. $/ 8$ )

$$
\kappa=\frac{1}{3} v \lambda
$$

The scattering mean free path is parametrized in terms of the $\mathrm{O}^{+}$ion gyroradius, $\lambda=\eta r_{c}$, where $\eta$ is assumed to be independent of $v$ and $x$. An altemative expression for $\lambda$ is

$$
\lambda=\eta \frac{\mathrm{mvc}}{\mathrm{eB}}
$$

where $m$ is the mass of the oxygen ion and $e$ is the electron charge. Substituting (6) into expressions (3) and (5) yields 


$$
\begin{gathered}
D=\frac{D_{0}}{\eta} \\
\kappa=\kappa_{0}\left(\frac{v}{v_{0}}\right)^{2} \eta
\end{gathered}
$$

where

$$
\begin{gathered}
D_{0}=\frac{3}{16 \pi} \frac{\mathrm{e}}{\mathrm{m}} \frac{\mathrm{B}^{3}}{\rho_{\text {ion }}} \\
\mathrm{x}_{0}=\frac{\mathrm{mcv}_{0}^{2}}{3 \mathrm{eB}}
\end{gathered}
$$

Equation (2) was numerically solved using an altemating direction fully implicit numerical scheme. It can be shown $/ 10 /$ that the second order accurate two-dimensional operator, $S_{x, y}(2 \Delta t)$, which integrates equation (2) from time $t$ to $t+2 \Delta t$, can be split into the following sequence of one-dimensional operators:

$$
S_{x, v}(2 \Delta t)=S_{x}(\Delta t) S_{v}(\Delta t) S_{y}(\Delta t) S_{x}(\Delta t)
$$

where $S_{x}$ and $S_{v}$ represent second order accurate one-dimensional time step operators.

\section{RESULTS AND DISCUSSION}

The model describes implanted heavy particle propagation upstream of the shock where $\rho_{i o n}=m_{p} n_{p}, m_{p}$ and $m_{p}$ being the proton mass and undisturbed solar wind number density. Adopting $B=10 \mathrm{nT}$ and $\mathrm{n}_{\mathrm{p}}=10 \mathrm{~cm}^{-3}$ for the undisturbed interplanetary magnetic field and solar wind number density values, we obtain $D_{0}=2.14 \times 10^{12} \mathrm{~cm}^{2} / \mathrm{s}^{3}$. Also, a reference velocity of $v_{0}=1340$ $\mathrm{km} / \mathrm{s}$ leads to $\mathrm{k}_{0}=10^{17} \mathrm{~cm}^{2} / \mathrm{s}$. Most simple spatial diffusion models use velocity independent diffusion coefficients, therefore we adopted an "average" spatial diffusion coefficient value of $x=k_{0} \eta$. The results presented in this paper were obtained with an $\eta$ value of 25 , which is consistent with observations $/ 3,4 \%$. In the calculations a $Q_{n}=10^{30}$ molecules/s gas production rate and a $\lambda_{n}=2 \times 10^{6} \mathrm{~km}$ ionization scale length was used.

The spatial variation of the plasma flow velocity was adopted from earlier self-consistent calculations 3,11 . The solar wind velocity was assumed to be $500 \mathrm{~km} / \mathrm{s}$ for cometocentric distances larger than $7 \times 10^{5} \mathrm{~km}$ (preshock region), it linearly decreased to $330 \mathrm{~km} / \mathrm{s}$ between $7 \times 10^{5} \mathrm{~km}$ and $3.8 \times 10^{5} \mathrm{~km}$ (foreshock). The shock itself was located between $3.8 \times 10^{5} \mathrm{~km}$ and $3 \times 10^{5} \mathrm{~km}$, where the flow velocity linearly decreased from $330 \mathrm{~km} / \mathrm{s}$ to $145 \mathrm{~km} / \mathrm{s}$. For cometocentric distances less than $3 \times 10^{5} \mathrm{~km}$ a linearly decreasing plasma bulk velocity was assumed (assuming zero flow velocity at $2 \times 10^{4} \mathrm{~km}$ ).

The calculation was carried out on a mesh of 200 spatial points by 140 velocity space points. The spatial grid covered the subsolar region from $0.22 \times 10^{6} \mathrm{~km}$ (downstream of the shock) to $4.22 \times 10^{6} \mathrm{~km}$ with a step size of $2 \times 10^{4} \mathrm{~km}$. The velocity grid extended from 0 to $3500 \mathrm{~km} / \mathrm{s}$ (corresponding to an $\mathrm{O}^{+}$energy of about $1000 \mathrm{keV}$ ) with a step size of $25 \mathrm{~km} / \mathrm{s}$. The boundarv conditions were $f(v=0)=0, f\left(v=v_{\max }\right)=0$ with free outflow boundaries at $x=x_{0}$ and $x=x_{\max }$. The initial condition was $f(x, v, t=0)=0$.

Fig. 2. shows the temporal evolution of the phase-space distribution function, F. Inspection of Fig. 2. reveals that velocity space diffusion very rapidly broadens the delta function like source. The mass addition always takes place at $v=u$, this effect can be easily seen at early times. At around 100 seconds the adiabatic acceleration starts to be noticeable at the shock. The characteristic time of the adiabatic acceleration is

$$
\frac{1}{\tau_{\text {adiabatic }}} \approx \frac{\mathrm{du}}{\mathrm{dx}}
$$

With our numerical values $\tau_{\text {diabetic }}=430 \mathrm{~s}$ at the shock and $2000 \mathrm{~s}$ in the foreshock region. As a result of the combined effect of velocity diffusion and adiabatic acceleration the velocity distribution becomes very broad by about 2000 seconds. The plasma convection time through our region is approximately $10^{4}$ seconds, therefore the phase-space distribution function reaches steadystate by about $2 \times 10^{4}$ seconds. For comparison it is interesting to calculate the characteristic time scales of first and second order Fermi accelerations. The time scale for change in velocity due to first order Fermi acceleration is /12/

$$
\tau_{\mathrm{F} 1}=\frac{4 \mathrm{~K}}{\mathrm{u}^{2}}
$$

From equations (3) and (5) one can calculate a comparable time scale for the second order Fermi process $/ 2,9 /$

$$
\tau_{F 2}=\frac{4 K}{V_{A}^{2}}
$$

Substituting our numerical values one obtains $\tau_{\mathrm{F} 1}=4 \times 10^{3}$ seconds and $\tau_{\mathrm{F} 2} \approx 2 \times 10^{5}$ seconds. However, it should also be noted that typical plasma transport time through the foreshock region (where the first order Fermi acceleration mechanism operates) is only about $10^{3}$ seconds, while there are millions of kilometers available (with a typical transit time of several times $10^{4} s$ ) for the second order Fermi acceleration in the far upstream region. 

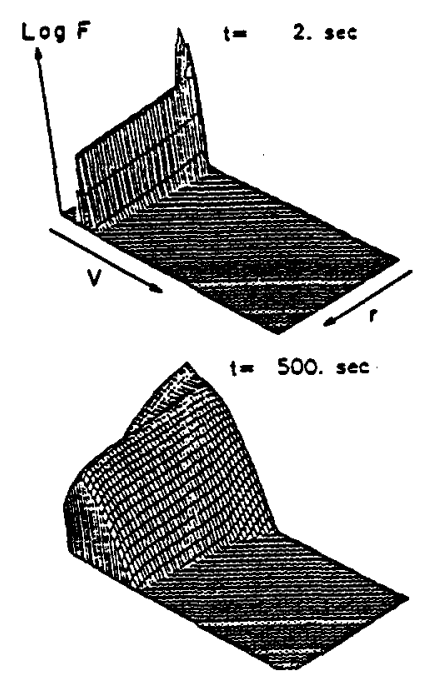
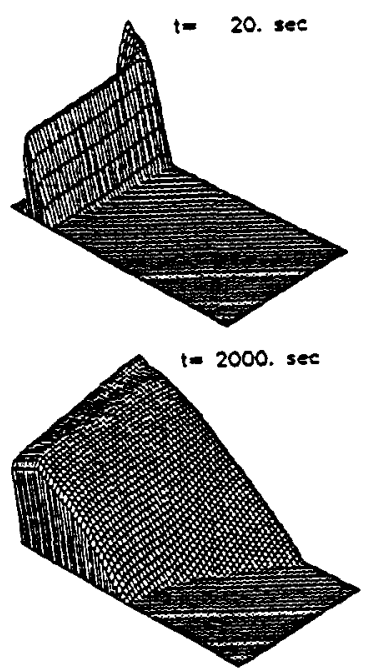

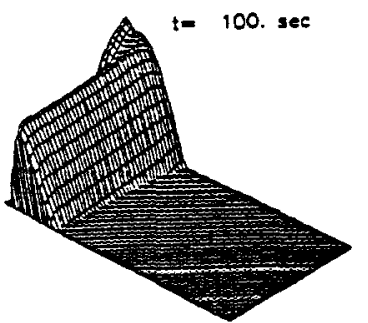

$t=20000$. see

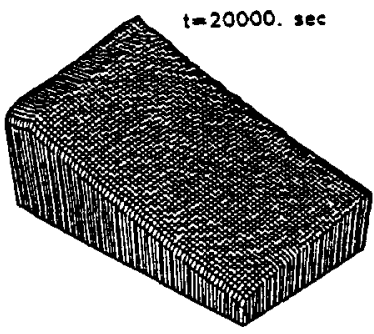

Fig. 2. Temporal evolution of the phase space distribution function, $F$.

Fig. 3. compares the relative importance of the various acceleration mechanisms. Fig. 3.a. shows the steady-state phase space density function, F, obtained by taking into account mass loading, convection and adiabatic compression (D=0, k=0). Fig 3.b. presents the same function obtained by solving the transport equation describing mass loading, convection, adiabatic compression and velocity space diffusion $(\kappa=0)$, while Fig. 3.c. shows the phase space distribution function obtained with mass loading, convection, adiabatic compression and spatial diffusion ( $D=0)$. Finally, Fig.3.d represents the distribution function when all of these processes operate together, ie. mass loading, convection, adiabatic compression, velocity and spatial diffusion. Inspection of Fig. 3. reveals that adiabatic compression alone is not adequate to produce energetic particles above $-1000 \mathrm{~km} / \mathrm{s}$ $(250 \mathrm{keV})$. On the hand adiabatic compression combined with either first or second order Fermi acceleration process may result in a sizable energetic particle population.

Detailed analyses of the solutions show that adiabatic compression combined with second order Fermi acceleration (Fig.3.b) results in exponential energy spectra near $100 \mathrm{keV}$ range with e-folding temperatures of about ten keV. This result is in good agreement with previous model calculations $/ 2,3 /$. On the other hand adiabatic compression combined with first order Fermi acceleration (Fig. 3.c.) results in power law type energy spectra with spectral indices between 12 and 15 . These spectra are considerably steeper than the ones observed at comets Halley /13/ or Giacobini-Zinner /14/.
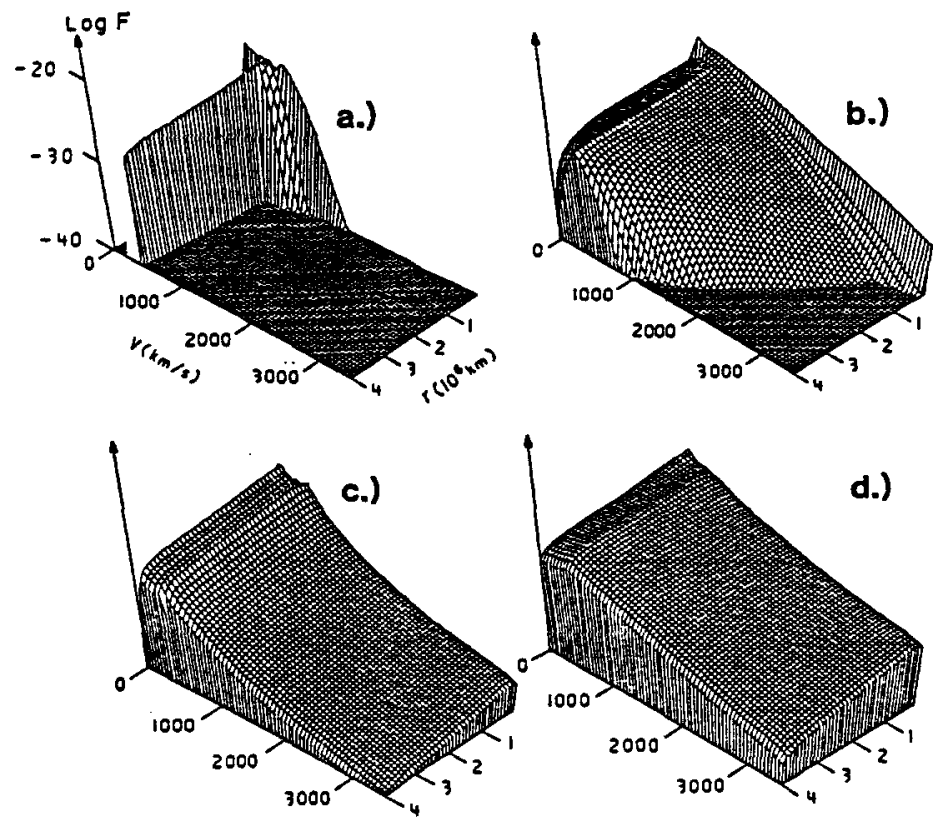

Fig. 3. Steady-state phase space distribution functions obtained with various acceleration processes.

a.) Adiabatic acceleration; b.) Adiabatic acceleration and velocity diffusion;

c.) Adiabatic acceleration and spatial diffusion; d.) Adiabatic acceleration, velocity and spatial diffusion. 
Finally, when both first and second order Fermi acceleration were operating (Fig. 3.d.) the steady-state distribution function reproduced the major observed features of the energetic particle population. Near the $100 \mathrm{keV}$ range the energy spectrum was reasonably well approximated by an exponential with e-folding temperatures in the $10 \mathrm{keV}$ range The energy spectra of the energetic particle population ( $>100 \mathrm{keV}$ ) can reasonably well approximated by power law spectra with spectral indices between 5.5 and 6. To illustrate this point Fig. 4. shows a typical energy spectrum just upstream of the bow shock $\left(4 \times 10^{5} \mathrm{~km}\right.$ from the comet). It can be seen that the high energy part of the spectrum follows a power law with an exponent of -5.5. Fig. 5. shows the spatial variation of the energy spectral index between $3.5 \times 10^{6} \mathrm{~km}$ and the bow shock. The calculated values are in a reasonably good agreement with the Giotto energetic particle observations /13/.

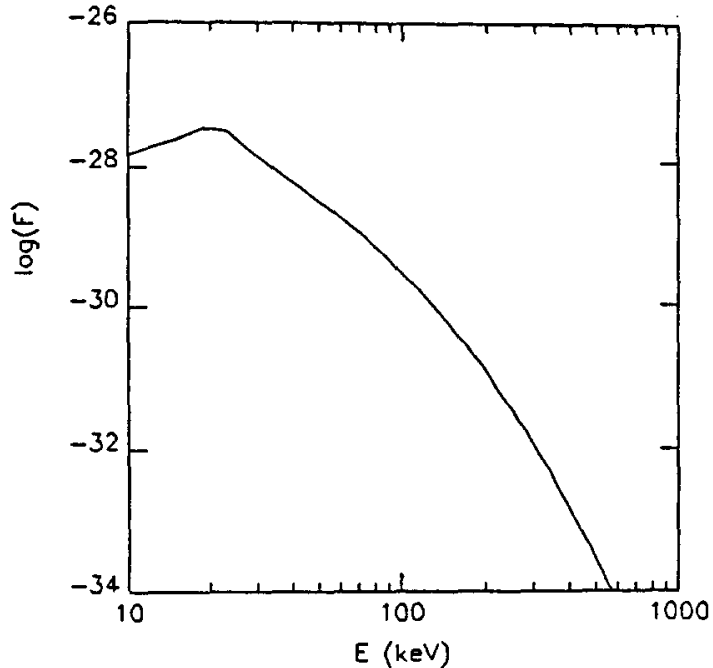

Fig. 4. Steady-state cometary ion energy spectrum at a cometocentric distance of $4 \times 10^{5} \mathrm{~km}$.

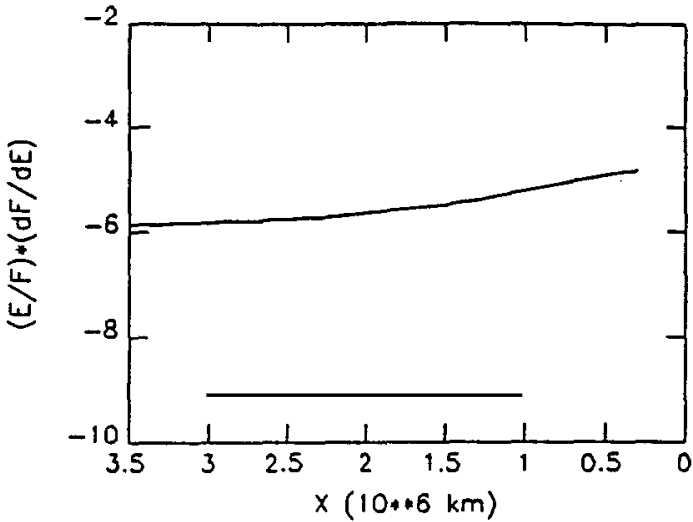

Fig. 5. Steady-state cometocentric distance dependence of the energy spectral index.

\section{ACKNOWLEDGEMENTS}

This work was supported by NSF grant AST-8605994 and NASA grant NAGW-1366 at the University of Michigan and by NASA grant NSG-7101 and NSF grant ATM-8618260 at the University of Arizona. Acknowledgement is also made to the National Center for Atmospheric Research sponsored by NSF, for the computing time used in this research.

\section{REFERENCES}

1. E. Amata and V. Formisano, Energization of positive ions in the cometary foreshock region, Planet. Space Sci, 33 , $1243(1985)$

2. W.-H. Ip.and W.I. Axford, The acceleration of particles in the vicinity of comets. Planet. Space Sci. 34, 1061, (1986)

3. T.I. Gombosi. Preshock region acceleration of implanted cometary $\mathrm{H}^{+}$and $\mathrm{O}^{+}, \mathrm{L}$ Géöphìs. Rés, 93,35 (1988)

4. W.-H. Ip, Cometary ion acceleration processes, Computer Physics Communicariöns, 49, 1 (1988)

5. T.I. Gombosi, J.R. Jokipii and K. Lorencz, First-ôrdêF Fêmí acceleration of pick-up ions in the cometary foreshock region, submitted to Geophys. Res. Lett. (1988)

6. A.J. Somogyi , K.I. Gñingaüz, K. Szzg̈o I. Szabo, Gy. Kozma, A.P. Remizov, J. Ero, I.N. Klimenko, I. T.Szilcs,M.I. Verigin.J. Windberg. T.E. Cravens, A.V. Dyachkov, G. Erdos, M. Farago, T.I. Gombosi, K. Kecskeméty, E. Keppler, T. Kovács, A. Kondor, Yu.I. Logachev, L. Lohonyai, R. Marsden, R. Redl, A.K. Richter, V.G. Stolpovskii, J. Szab6, I. Szentpétery, A. Szepesváry, M. Tátrallyay, A. Varga, K.P. Wenzel, G.A. Vladimirova and A. Zarandy, First observations of energetic particles near comet Halley, Nanure, 321, 285 (1986)

7. H.U. Schmidt, and R. Wegmann, Plasma flow and magnetic fields in comets, in: Comets, ed. L.L. Wilkening, Univ. Arizona , Tucson 1982, p. 538.

8. J.R. Jokipii, Propagation of cosmic rays in the solar wind, Rey, Geophys. Soace Phys. 9, 27 (1971)

9. L.A. Fisk, On the acceleration of energetic particles in the interplanetary medium, L. Geophys. Res, 81,4641 (1976)

10. A.R. Gourlay and J.L. Morris, L. Comp. Phys, 5, 229 (1970)

11. T.I. Gombosi, Charge exchange avalanche at the cometopause, Geophys. Res. Lett, 14, 1174 (1987)

12. Drury, L., An introduction to the theory of diffusive shock acceleration of energetic particles in tenuous plasmas, Rept. Prog. Phys. 46, 973 (1983)

13. D. O'Sullivan, A. Thompson, S. McKenna-Lawlor, P.W. Daly, E. Kirsch and K.P. Wenzel, Observations of the comet Halley foreshock region by the EPA instrument on board the Giotto spacecraft, this issue.

14. I.G. Richardson, S.W.H. Cowley, R.J. Hynds, C. Tranquille, T.R. Sanderson, K.P. Wenzel and P.W. Daly, Planet. and Space Sci, 35, 1323 (1987) 